HIV-ASSOCIATED

CARDIO-

MYOPATHY

\title{
The hidden continuum of HIV- associated cardiomyopathy: A focussed review with case reports
}

Pieter-Paul S. Robbertse", Anton F. Doubell", Jean B. Nachega $a^{\#+,+*}$ and Philip G. Herbst*

"Division of Cardiology, Department of Medicine, Faculty of Medicine and Health Sciences, Stellenbosch University and Tygerberg Academic Hospital, Tygerberg, South Africa "Department of Medicine and Centre for Infectious Diseases, Faculty of Medicine and Health Sciences, Stellenbosch University, Cape Town, South Africa

Department of Epidemiology and International Health, Johns Hopkins Bloomberg School of Public Health, Baltimore, Maryland, United States of America

*Department of Epidemiology, Infectious Diseases and Microbiology, and Centre for Global Health, University of Pittsburgh, Pittsburgh, Pennsylvania, United States of America

Address for correspondence:

Dr Pieter-Paul S. Robbertse

Division of Cardiology

Department of Medicine

University of Stellenbosch and Tygerberg Academic Hospital

PO Box 19063

Tygerberg

7505

South Africa

\section{Email:}

psrobbertse@gmail.com

\section{INTRODUCTION}

HIV associated cardiomyopathy (HIVAC) has a poorly understood pathology

Human immunodeficiency virus (HIV) infection and cardiovascular disease (CVD) are intimately related, and a leading cause of death and disability worldwide. ${ }^{(1,2)}$ Previous literature emphasises the variety of CVD encountered in people living with HIV (PLWH). ${ }^{(3)}$ However, the coronary disease burden seen in high-income countries contrasts with what is experienced in low- and middle-income countries ${ }^{(3,4)}$ where the vast majority of PLWH reside. ${ }^{(5)}$ In these resource-limited countries, myocardial disease remains prevalent. (4,6,7) $^{-}$Cardiomyopathy is a feared HIV-complication with a poor prognosis once systolic dysfunction is present. ${ }^{(8)}$ Although the rollout of anti-retroviral

\section{ABSTRACT}

HIV-associated cardiomyopathy (HIVAC) is a poorly understood group of diseases with a poor prognosis once ventricular dysfunction is present. Cardiovascular magnetic resonance has revealed a previously unappreciated burden of asymptomatic myocardial abnormalities in people living with HIV, including abnormalities already present at the time of HIV diagnosis. These abnormalities include thickened, inflamed ventricles that bear resemblance to cases of symptomatic HIVAC that are reported on in this article. Our understanding and the significance of asymptomatic HIV-associated myocardial pathology will be explored as early disease on a continuum towards more advanced cardiomyopathy. The need for prospective research in persons naïve to anti-retroviral therapy is emphasised as it may provide key findings to better understand this elusive disease process. SAHeart 2021;18:126-135

treatment (ART) has made HIVAC less common and improved outcomes, HIV infection remains a major risk factor for cardiac dysfunction. ${ }^{(3)}$ There is currently no universally accepted terminology for HIVAC. HIVAC is a collective term used to describe a number of cardiomyopathic findings that may not have a clear cause. It is helpful to broadly categorise HIVAC into 3 groups: i) Myocarditis; ii) Asymptomatic diastolic or systolic ventricular dysfunction; and iii) Symptomatic dilated cardiomyopathy with reduced systolic function. ${ }^{(9)}$ Treatment is challenging due to the poorly understood pathophysiology and the lack of clinical trials to provide evidence for the tailored management of these patients. It is unclear if current guideline directed medical therapy is the best option to optimise long term outcomes. Furthermore, it remains unknown which treatments supplementing ART are required to adequately prevent and treat HIVAC. ${ }^{(6)}$

This narrative review will highlight similarities between symptomatic and asymptomatic HIV associated myocardial pathology as observed with cardiovascular magnetic resonance (CMR). Our understanding and the significance of myocardial pathology in asymptomatic PLWH will be explored as early disease on a continuum towards more advanced disease. Furthermore, 
the need for prospective research in persons naive to ART will be discussed as it may provide clues to a better understanding of this elusive disease process.

\section{THE TOOL OF CHOICE - CMR AND VIRTUAL HISTOLOGY}

To explore underlying pathological processes of disease, histology-based research has long been the gold standard. Unfortunately, this form of investigation is limited by the invasive nature of antemortem tissue biopsies or necropsies.

Echocardiography has been the workhorse modality for evaluating cardiac structure and function in the clinical context. Unfortunately, this modality requires significant numbers to discern differences between groups in the study context due to modest inter- and intra-observer variability. ${ }^{(10)}$ This becomes relevant when noting that echocardiography studies assessing myocardial disease mechanisms in PLWH remain relatively small. The use of strain-imaging improves echocardiography's ability to detect functional abnormalities of the myocardium. This modality has been used to study PLWH and has demonstrated subtle myocardial abnormalities that are present well before symptoms, biochemical evidence, or conventional echocardiographic findings of myocardial dysfunction.( ${ }^{(1)}$ Unfortunately, strain-abnormalities remain non-specific as they only provide functional information on myocardial contraction mechanics and deformation. ${ }^{(12,13)}$ Strain-imaging cannot characterise tissues and is therefore unable to obtain direct evidence of pathological processes that underlie myocardial dysfunction.

CMR allows for comprehensive, serial evaluation of cardiac structure and function. Evaluation of pathology through a virtual histology approach is a unique feature of CMR. This allows for non-invasive examination of pathological processes and how they interact with the observed cardiac structure and function in vivo. It is an excellent tool to non-invasively obtain information on myocardial tissue at varying clinical stages of disease. These include the presence of inflammation, oedema, necrosis, fibrosis, infarction, infiltration and thrombosis. ${ }^{(14,15)}$ Non-invasive measurement of tissue characteristics have led to the development of CMR-biomarkers. T1, T2 and T2* (T2 star) are magnetic resonance imaging (MRI) measurements that represent different tissue relaxation parameters following excitation by a radiofrequency pulse. ${ }^{(16)}$ Different tissues have unique $T$ I, $\mathrm{T} 2$ and $\mathrm{T} 2$ * properties that can be used to demonstrate specific tissue characteristics or pathological processes in a magnetic resonance image. These imaging biomarkers may be used to support the diagnosis, to prognosticate disease, and for surveillance during treatment. ${ }^{(17)}$ Examples include T2* measurement to guide iron chelation therapy in thalassemia and haemochromatosis ${ }^{(18)}$ and TI/extracellular volume (ECV) measurement to monitor amyloidosis treatment. ${ }^{(17)}$ Limited studies evaluating the myocardial tissue characteristics of PLWH have been published. ${ }^{(19-21)} \mathrm{A}$ number of observations from the CMR scans of PLWH offer insight into the direction that may be taken to shed light on the underlying myocardial pathology.

\section{CASE REPORTS}

Two case reports are presented demonstrating the evolution of symptomatic HIVAC as studied by CMR. These cases will be utilised as a point of departure and substrate for discussion in this article.

\section{Case I}

A 27-year-old female with no prior cardiovascular disease or symptoms, presented with an embolic stoke. She was known to be HIV-infected, but naive to ART with a CD4 count of 6 I cells/microlitre. She denied smoking, excessive alcohol consumption or injection drug use. No nutritional deficiencies were suspected. She presented 2 years before the worldwide coronavirus disease 2019 (COVID-19) outbreak. Abnormalities seen on echocardiography prompted a CMR study with key findings shown in Figure I, left panel. Left ventricular (LV) systolic function was impaired with an ejection fraction of $48 \%$ (see online supplement video clip). The LV was non-dilated, suggesting an acute/subacute process. Indexed LV end diastolic volume measured $64 \mathrm{ml} / \mathrm{m}^{2}$. Furthermore, the LV walls appeared thickened. The LV mass index measured high normal at $72 \mathrm{~g} / \mathrm{m}^{2}$. Myocardial oedema was demonstrated by an increased signal on short tau inversion recovery (STIR) images. These T2-weighted images are designed to demonstrate high signal in tissues with an increased water content (oedema). ${ }^{(16)}$

These findings were consistent with a frequently encountered myocardial phenotype seen in PLWH, namely poorly functional ventricles with a high LV mass index. This phenomenon of heavier ventricles has been explored using echocardiography in asymptomatic PLWH and is independently associated with HIV infection. ${ }^{(22)}$

Oedema as part of an inflammatory process in the myocardium was the most likely explanation for the CMR findings. An endomyocardial biopsy (EMB) confirmed myocarditis, with the viral panel screening negative for parvovirus $B \mid 9$, respiratory viruses and enteroviruses. Serology for Hepatitis B virus was negative. Autoimmune workup, including anti-nuclear 

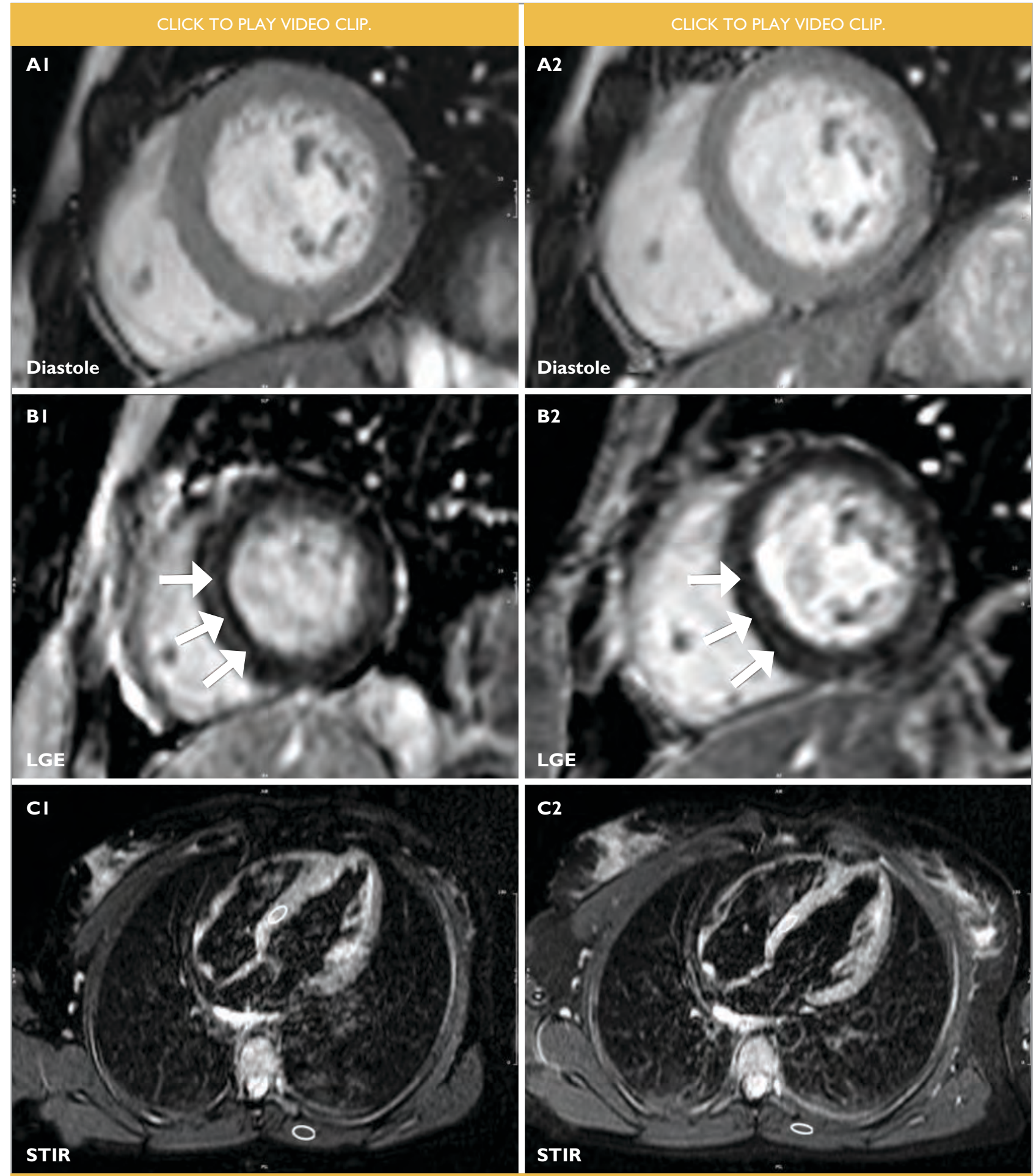

FIGURE I: Comparative findings in an HIV positive female with HIV-associated cardiomyopathy before (I, left panel) and 9 months after initiation of antiretroviral treatment (2, right panel).

(A) basal short axis, end diastolic frames of cine imaging. Left ventricular ejection fraction at baseline measured $48 \%$ and improved to $59 \%$ on treatment. Indexed left ventricular end diastolic volume at baseline and follow-up were $64 \mathrm{ml} / \mathrm{m}^{2}$ and $72 \mathrm{ml} / \mathrm{m}^{2}$ respectively. The left ventricular mass index improved from a baseline value of $72 \mathrm{~g} / \mathrm{m}^{2}$ to $56 \mathrm{~g} / \mathrm{m}^{2}$.

(B) basal short axis, phase sensitive inversion recovery images showing midmyocardial late gadolinium enhancement (LGE) of the septum at baseline and follow-up (white arrows).

(C) 4-Chamber, short tau inversion recovery (STIR) image showing myocardial: skeletal signal ratio (white circles) of 2.6, indicative of ventricular oedema at baseline $(\mathrm{CI})$. STIR imaging at follow up (C2) demonstrated myocardial: skeletal signal ratio (white circles) of 2.6, suggesting ongoing ventricular oedema despite antiretroviral treatment. 
antibodies, was negative. The etiology therefore remained largely unexplained.

Follow-up CMR-evaluation allowed the prospective study of disease evolution after the initiation on ART. At the time of follow up, the CD4 count had improved to 172 cells/microlitre, despite the patient not achieving viral suppression (viral load = 252405 copies $/ \mathrm{ml}$ ). Within a relatively short period of time ( 9 months on ART), the LV ejection fraction improved to $59 \%$ and the left ventricular mass index decreased to $56 \mathrm{~g} / \mathrm{m}^{2}$. The indexed LV end diastolic volume remained fairly similar at $72 \mathrm{ml} / \mathrm{m}^{2}$. The longitudinal systolic function (not shown) did not fully recover and evidence of persistent myocardial inflammation was present (Figure I, C2).

The observation of ongoing myocardial inflammation despite treatment supports contemporary literature on myocardial pathology in PLWH and will be discussed later in the article. The persistence of late gadolinium enhancement (LGE) in the septum supports the presence of myocardial fibrosis and is consistent with the pattern of LGE seen in dilated cardiomyopathies, irrespective of aetiology. ${ }^{(18)}$

LGE is currently the reference standard for non-invasive demonstration of myocardial scar and focal fibrosis in both ischaemic and non-ischaemic pathologies.(17) LGE is most notably associated with myocardial necrosis and fibrosis, but may be present with ventricular oedema if the degree of interstitial expansion is large enough. ${ }^{(23)}$ Gadolinium-based contrast agents do not cross intact cell membranes and therefore distribute exclusively in the extracellular space. After intravenous administration, gadolinium is trapped in expanded interstitium, lowering the TI time of these abnormal tissues. The wash out time of gadolinium in tissues with an expanded interstitium is longer than normal tissue. This increases the signal during delayed enhancement imaging and allows for improved contrast between normal and abnormal tissues.(24) Interstitial oedema, replacement fibrosis and myocardial necrosis are all causes of interstitial expansion.(18) The degree of interstitial expansion from oedema is typically less than with replacement fibrosis and necrosis. This gives a picture of less intense gadolinium signal ("bland gadolinium") as opposed to the brighter signal typically seen in infarction and replacement fibrosis. Interstitial expansion from oedema may however be marked when an associated cellular infiltrate is present. ${ }^{(25)}$ As an extracellular contrast agent, the gadolinium will only occupy the non-cellular component of the expanded extracellular space. As such, the cellular infiltrate acts to "dilute" the gadolinium in this total expanded space leading to a less intense gadolinium signal. Therefore, "bland gadolinium" may be encountered despite marked interstitial expansion where a large fraction of this is related to cellular infiltrates. The intensity of LGE may decrease between CMR studies if the degree of oedema has improved sufficiently without accompanying fibrosis. (23)

LGE imaging is an excellent modality to diagnose focal myocardial abnormality as seen in replacement fibrosis. The interpretation of LGE findings may, however, be challenging when global myocardial pathology is present. This includes diffuse myocardial fibrosis as is frequently encountered in common pathologies including hypertensive heart disease and aortic stenosis. Furthermore, global myocardial oedema may be present in pan-myocarditis associated with systemic diseases such as systemic lupus erythematosus, rheumatoid arthritis and likely HIV. (26) This is due to the fact that LGE assessment is usually a qualitative evaluation and involves comparison with an area of normal myocardium by the observer. If global, homogenous LGE of the myocardium is present, comparison with a normal myocardial reference area is not possible. It is therefore possible that LGE may be missed in these scenarios. Tools used to quantify LGE have been developed. Like visual LGE assessment, LGE quantification is dependent on an area of normal myocardium that serves as a reference against which abnormality is assessed. (17) When global LGE is present, the same problem is faced as with visual assessment - no normal myocardial reference is possible and measurement accuracy is decreased. Furthermore, the use of gadolinium-based contrast agents may be contra-indicated in patients with severe renal disease as these agents have been associated with rare cases of nephrogenic systemic fibrosis.(23) The above challenges can be overcome by employing CMR's myocardial mapping techniques. ${ }^{(17)}$

Myocardial mapping sequences are gaining popularity to study focal and global myocardial processes and have distinct advantages over LGE imaging. These robust imaging sequences allow for quantitative evaluation of the myocardium without the need for a normal reference region. Another advantage of mapping sequences is that they do not generally require gadolinium administration and are safe to use in patients at higher risk for nephrogenic systemic fibrosis. These sequences allow for serial evaluation and comparison of quantitative imaging biomarkers TI, T2, T2* and ECV.

Mapping sequences were employed to prospectively study a patient with symptomatic HIVAC demonstrating the utility of these sequences to compare imaging biomarkers over time. 


\section{Case 2}

A 38-year-old male presented with a one-week history of symptoms in keeping with left sided cardiac failure following a flu-like illness. He did not report any prior illness, excessive alcohol consumption or injection drug use. He appeared chronically unwell with generalised body wasting and a body mass index of $19 \mathrm{~kg} / \mathrm{m}^{2}$. No evidence of specific nutritional deficiencies were present. He was diagnosed with HIV at presentation and had a CD4 count of 208 cells/microlitre. An echocardiogram demonstrated marked structural and functional abnormalities. A CMR study was performed at the time of presentation to assist workup (Figure 2). The LV was dilated and had a severely reduced ejection fraction of $26 \%$ (see online supplement video clip). The ventricular walls were markedly thickened with a maximum septal measurement of $17 \mathrm{~mm}$. Indexed LV end diastolic volume and mass measured $153 \mathrm{ml} / \mathrm{m}^{2}$ and $180 \mathrm{~g} / \mathrm{m}^{2}$ respectively - both notably raised. Overt myocardial oedema was present as evidenced by high $\mathrm{TI}$ and $\mathrm{T} 2$ mapping values, as well as a global average STIR myocardial: skeletal muscle ratio of 2.5 (not shown). An endomyocardial biopsy confirmed myocarditis with no causative organism identified on the viral panel. The panel included screening for parvovirus B 19 , respiratory- viruses, herpes-, and enteroviruses. Serology for Hepatitis B virus was negative. Autoimmune workup, including anti-cardiac antibodies, was negative.

A follow up CMR was performed 4 months after initiation on ART and anti-failure treatment (Figure 2, right). The patient had an undetectable viral load at this time. The LV ejection fraction had improved from $26 \%$ - $45 \%$ on treatment. The indexed LV end diastolic volume and LV mass index improved to $119 \mathrm{ml} / \mathrm{m}^{2}$ and $158 \mathrm{~g} / \mathrm{m}^{2}$, respectively. Septal thickness remained unchanged at $17 \mathrm{~mm}$. Global average STIR myocardial: skeletal muscle ratio decreased to 1.94, which is borderline for myocardial oedema.

In the absence of other aetiologies, these two HIV-positive patients demonstrate clear symptomatic myocardial pathology. The myocardial involvement of case 2 is significantly more advanced than case one, but shared features of chronic myocarditis are evident: poorly contractile, oedematous ventricles that improve, but do not normalise on ART. Persistent myocardial inflammation was observed in both cases, irrespective of whether viral suppression was achieved or not. The aetiology of their cardiomyopathies remains elusive and forces us to confront our incomplete understanding of myocardial pathology in HIV. These cases give us the opportunity to demonstrate the effects of ART on symptomatic myocardial disease over time using CMR. This strategy will prove invaluable in future research to disentangle a complex, multifactorial disease process.

\section{THE PATHOLOGY OF HIVAC - KEY KNOWLEDGE GAPS REMAIN}

The pathophysiology of HIVAC is complex and has proven to be difficult to research. Various hypotheses have been proposed and involve chronic inflammation secondary to immune activation, post-infective cardiac autoimmunity, primary or secondary HIV myocarditis, immunodeficiency, opportunistic infections, complications of ART, nutritional deficiencies, traditional risk factors, as well as alcohol consumption and smoking. $\left.{ }^{6}, 9,14,19,27-33\right)$ It is not known whether a genetic predisposition to HIVAC exists and limited research has been performed in this field. ${ }^{(34)}$

In practice, the cause is most probably multifactorial and likely to represent a complex interplay of one or more of these processes. Chronic myocardial inflammation appears to be a key finding and cumulative myocardial damage from inflammation has been proposed.(19) Inflammation may be a common pathophysiological endpoint of various processes that work synergistically to create the perfect storm for myocardial damage (Figure 3). The relative contribution of these factors may vary depending on the setting, as different causes have been noted between high-income and low-income countries, as well as virologically supressed and unsuppressed individuals. ${ }^{(6)}$ The influence of ART on the reversibility of myocardial abnormalities that are already present remain largely unstudied. Current evidence suggests that even modern ART is not the sole answer to prevent and treat HIVAC. ${ }^{(19)}$ Many questions remain unanswered, including to what degree viral suppression protects the myocardium from disease progression and during what stage of HIV infection myocardial pathology starts to manifest. Further research is clearly required.

With the advent of more accessible ART worldwide, the focus of research on CVD in PLWH has begun to shift. An area of interest is the study of asymptomatic PLWH.

\section{THE BLACK BOX}

Do all PLWH develop some degree of asymptomatic myocardial involvement yet only some progress to symptomatic cardiomyopathy?

A number of authors have investigated asymptomatic myocardial pathology in PLWH using various modalities. Findings have shown a previously underappreciated burden of subclinical cardiomyopathy. Pre-ART era research from a lowincome, African country observed dilated cardiomyopathy using echocardiography in $17.7 \%$ of asymptomatic PLWH. (35) Similarly, an American group prospectively performed echo- 

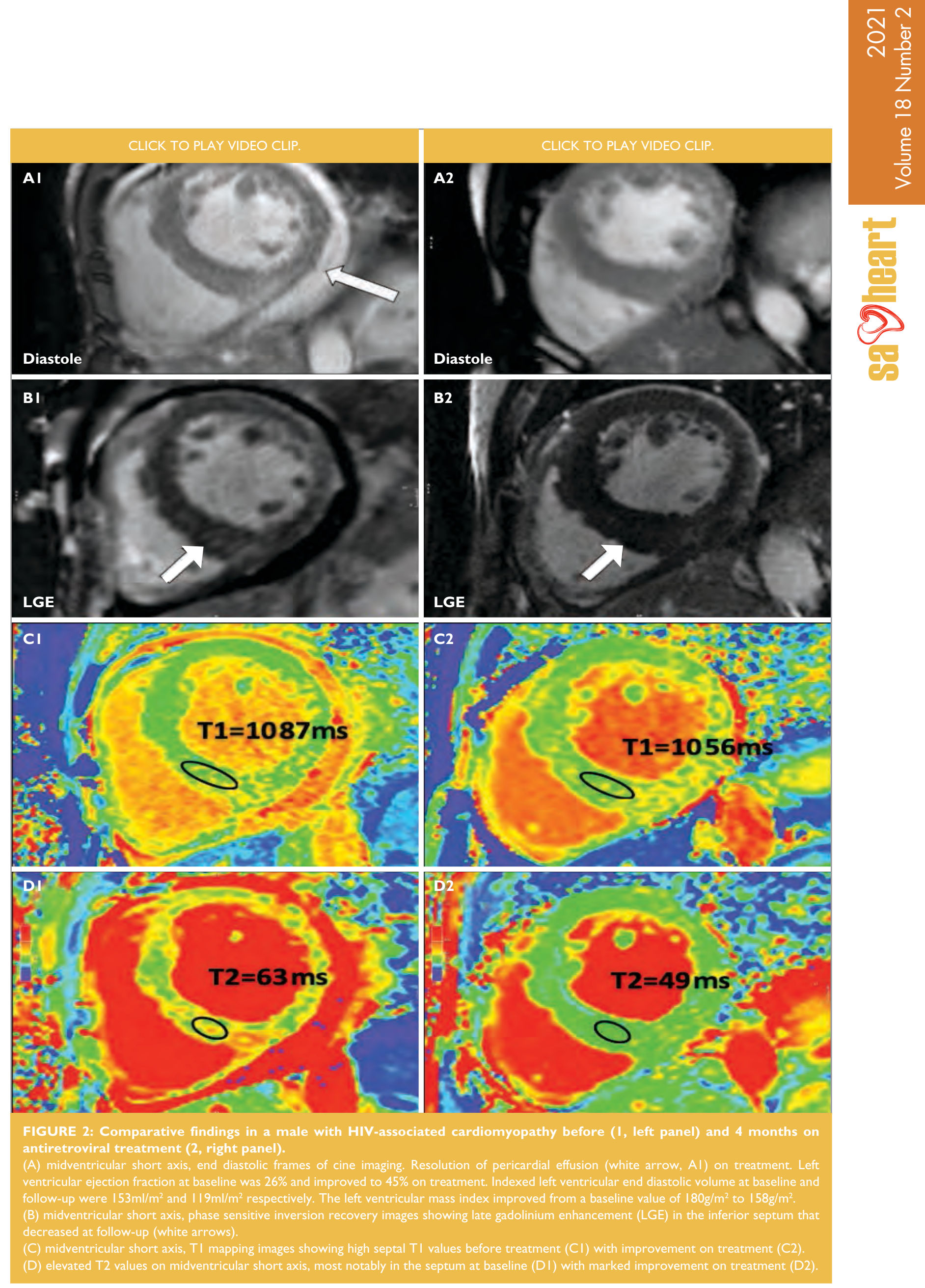
cardiograms on 69 newly diagnosed HIV positive patients not suspected of having cardiovascular disease. Unexpectedly, 14.5\% had myopathic ventricles at baseline. During an IImonth follow-up period, $18.6 \%$ of the participants with normal hearts at baseline subsequently developed echocardiographic evidence of cardiomyopathy in the absence of ART. The prevalence of HIVAC in this cohort was as high as 30.4\%.(36) $\mathrm{Had}$ current CMR technology been employed to study these individuals, it is not unreasonable to speculate that the prevalence of myocardial abnormalities may have been even higher. Furthermore, this study demonstrated the progression of HIVAC in a large proportion of patients with the only demonstrable risk being a CD4 count of less than 100 cells/ microlitre. No meaningful association with alcohol abuse, smoking, diabetes, an AIDS diagnosis, or diastolic hypertension were demonstrable. A trend of higher rates of cardiomyopathy in patients older than 40 years was present but did not prove statistically significant.

A Brazilian autopsy/histopathology-based study investigated 94 patients with AIDS in the absence of ART. Varying degrees of cardiac dilatation was described in $74 \%$ of necropsies. The macroscopically remodelled hearts were not related to any known cardiovascular disease. When investigating the hearts microscopically, a variety of pathological cardiac alterations were present in almost all cases. These findings included myocardial alterations in the form of interstitial fibrosis in $45.5 \%$ of cases and interstitial mononuclear infiltrate in all cases. On ultrastructural level, interstitial oedema was observed in all cases, with cardiomyocyte apoptosis present in $44 \%$ of the cohort. ${ }^{(37)}$ Cardiac steatosis was a rare finding, with only mild steatosis reported in $4.5 \%$ of cases.

These findings emphasise a clear disconnect in the literature: PLWH have almost universal underlying myocardial pathology, yet only some individuals develop symptomatic disease. This observation leaves the impression that myocardial involvement is on a continuum of disease, ranging from trivial inflammation in otherwise normal hearts to a poorly prognostic dilated cardiomyopathy phenotype. What happens in a real world setting between these two extremes remains unknown. Patients with subtle, largely undiagnosed myocardial abnormalities receive ART and enter the "Black Box" (Figure 3). Years later we are faced with a subset of patients who developed symptomatic cardiomyopathy or are found to have asymptomatic cardiomyopathy with no clear idea as to what happened in the interim. Identifying patients at greatest risk for progression, predictors of disease attenuation and the most effective surveillance and treatment modalities remain unclear.
To develop strategies to answer the above questions, researchers need to establish a timeline for HIVAC which describes the disease phenotype at different time points in its progression.

Holloway, et al. utilised CMR and spectroscopy to study asymptomatic PLWH already on ART in the United Kingdom. ${ }^{(21)}$ The authors demonstrated altered myocardial functioning, myocardial steatosis and fibrosis in their participants. The high rate of cardiac steatosis is thought to be the result of ARTrelated metabolic derangement. The low prevalence of steatosis in autopsy-studies in pre-ART era AIDS patients ${ }^{(37)}$ supports the theory of ART induced myocardial toxicity. Investigating persons on ART may further confound the interpretation of findings, as this group already has various potential mechanisms of myocardial damage. In order to disentangle these various processes, the need for work on ART naive individuals has been emphasised.

Appraising CMR-publications that studied asymptomatic, ARTnaive individuals, myocardial abnormalities appear to be present before the initiation of ART. The work of Holloway, et al. was expanded upon by Ntusi, et al.(19) The enlarged cohort included $13 \mathrm{PLWH}$ naive to ART and sought to compare myocardial inflammation and oedema in various patient groups, including HIV uninfected controls. Compared to treated individuals, the ART naive subgroup demonstrated decreased left ventricular ejection fraction. Treated and untreated individuals had equally high rates of myocardial LGE (82\% vs. $85 \%$ ). LGE was most frequently visualised in the septum, posterior, and lateral segments of the base and mid-ventricle. Compared to HIV uninfected controls, the ventricular mass and oedema markers were significantly increased in both treated and untreated PLWH.

Findings from the high- to middle-income country of Peru were briefly discussed in a letter to the editor by Menacho, et al.(20) The authors report similar findings of subtle reduced systolic functioning and high rates of LGE in untreated and treated PLWH. The pattern of LGE was similar to what was seen by Ntusi, et al.: septal and posterior segment involvement. Notable findings in this group are a global increase in native TI, T2 and ECV found in PLWH. Unfortunately, no data on LV mass was presented for comparison.

The findings of Holloway, et al., Ntusi, et al. and Menacho, et al. all mirror the observations in symptomatic cardiomyopathy presented in cases I and 2 (Figures I and 2): Thickened, inflamed ventricles that incompletely improve on ART. Although the 


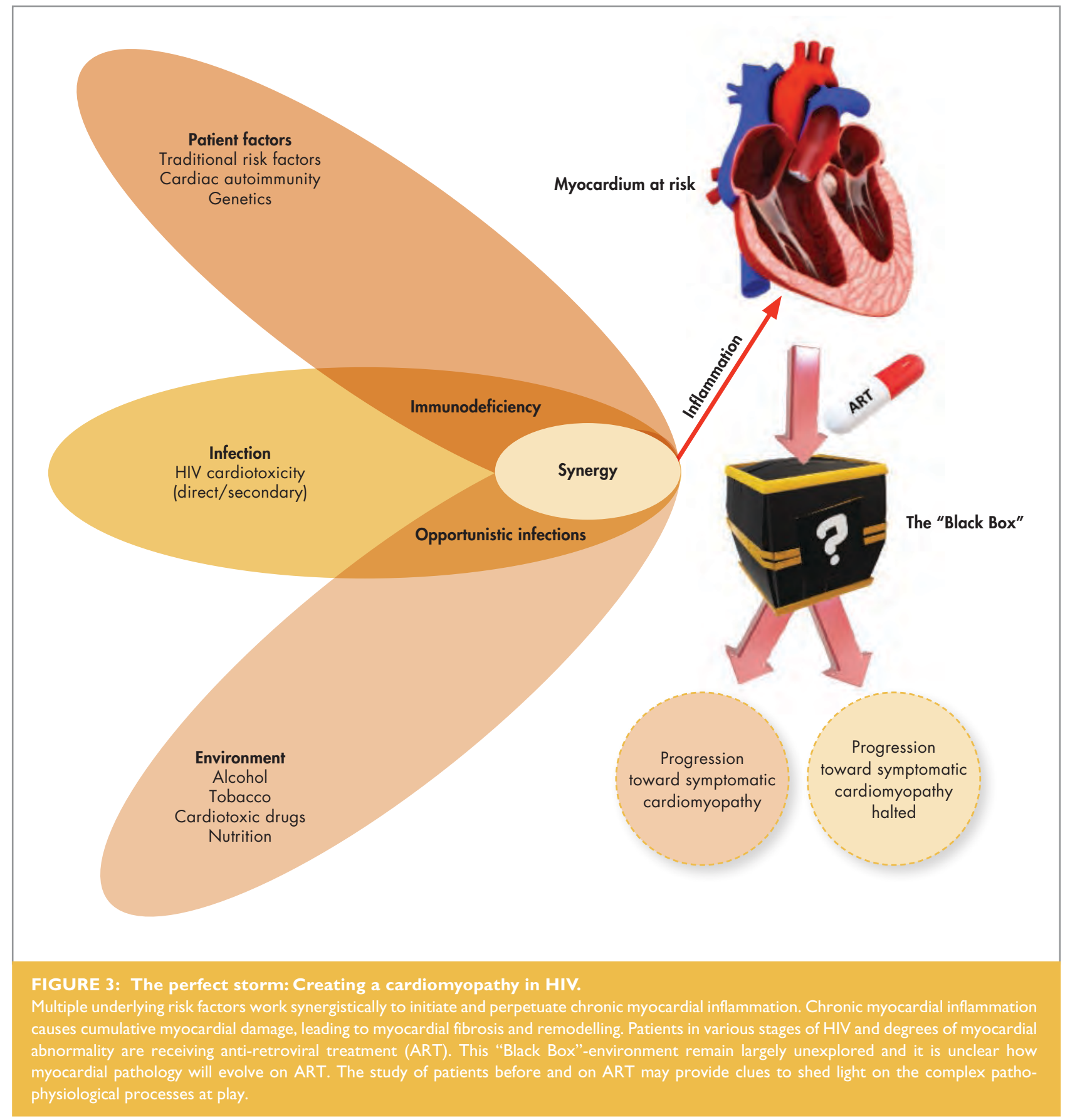

similarity of symptomatic and asymptomatic cases are striking, the degree to which subclinical myocardial abnormality is a harbinger of more advanced pathology, and to what extent ART will improve or halt evolution of abnormalities remains unknown. ${ }^{(28)}$

A recent biochemical study has provided evidence to suggest that an inflammatory cardiomyopathy is present at the time of HIV-seroconversion. It was demonstrated that subclinical cardiac dysfunction and myocardial damage are present during acute HIV infection, and improve with viral suppression. (38) NTproBNP was used as a surrogate for ventricular functioning while troponin $T$ represented myocardial injury. NT-proBNP and troponin $T$ showed significant correlation with the plasma viral load. The authors speculate that the inflammatory surge, high HIV viral load, and immunosuppression experienced during acute HIV infection, may be the cornerstone of progressive myocardial disease. This represents the earliest possible time point that HIV may affect the myocardium. Unfortunately, no imaging was performed in this study, and it would have been 
enlightening to correlate these findings with observed cardiac functioning and CMR's virtual histology.

A unifying hypothesis would be that we are observing snapshots of the same disease process over time on a continuum towards dilated, poorly functional ventricles. Myocardial inflammation appears to be a common end-point for various driving processes. Prospective research is lacking to provide additional evidence for this hypothesis.

Another shortcoming of currently available literature is the underrepresentation of the developing world in research. Cohorts are dominated by older, Caucasian males that do not live in the same environment as the majority of PLWH; namely younger, African females in low- to middle-income countries. ${ }^{(5)}$ The spectrum of cardiovascular disease is reliant on the specific population group and their environment.(6) Differences that might plausibly change observed cardiovascular disease include host genetics, prevalent co-infections and their modulatory effect on the immune system, as well as delayed presentation and treatment in resource limited settings. These factors may further limit our understanding of the already complex processes at play.

Research evaluating asymptomatic, ART naive persons in the developing world will provide a novel perspective on HIVAC. It is possible that the underlying burden of cardiomyopathy differs significantly from data predominantly generated in the developed world. Furthermore, future study in this field should aim to better represent non-Caucasian people and females. The identification of abnormal tissue characteristics in asymptomatic, ART naive PLWH will allow for prospective, serial evaluation with CMR after initiation on ART.

\section{CONCLUSION}

HIVAC is an incompletely understood disease-group with a poor prognosis once established ventricular dysfunction is present. The pathophysiology is complex with multiple processes likely working synergistically to set in motion and maintain a state of chronic myocardial inflammation. In turn, chronic inflammation drives cardiac fibrosis and remodelling.

An overlap of ventricular inflammation, oedema and fibrosis is demonstrable in symptomatic and asymptomatic persons with HIVAC. This phenomenon, together with evidence of a high burden of early myocardial involvement in PLWH, creates the impression that we are observing the same pathology at different time points on a continuum from asymptomatic myocardial inflammation towards end stage dilated cardiomyopathy.

Serial evaluation using CMR's virtual histology approach is an exciting tool to investigate HIVAC. This prospective approach will enable us to peek into the previously inaccessible "Black Box" and observe real time pathology. Information on the evolution of myocardial abnormalities on ART is needed. Current $C M R$ evidence suggests the concerning notion that ART alone does not fully address cardiovascular inflammation associated with HIV infection. As the authors have explored, most, if not all, PLWH appear to have some degree of underlying myocardial abnormality. It is unclear what subset of these patients will continue to progress towards end-stage cardiomyopathy. The ability to accurately predict this will only be made possible by improving our understanding of the disease process.

\section{ACKNOWLEDGMENTS}

Dr Pieter-Paul S. Robbertse and Professor Jean B. Nachega are supported by the United States National Institutes of Health (NIH)/Fogarty International Centre (FIC) grant No. I D43TW0 I0937-0 I AI (University of Pittsburgh HIV Comorbidities Research Training Program in South Africa - Pitt-HRTPSA). We acknowledge critical review by Peter Hunt, M.D., University of California San Francisco (UCSF), San Francisco, CA, USA. We thank Dr Karim M. Hassan from the Division of Cardiology, Tygerberg Academic Hospital for performing the workup and endomyocardial biopsies of the clinical cases.

\section{Conflict of interest: none declared.}


I. Smith CJ, Ryom L, Weber R, et al. Trends in underlying causes of death in people with HIV from 1999 - 201 I (D:A:D): A multicohort collaboration. Lancet. 2014;384:24I-248.

2. Erqou S, Lodebo BT, Masri A, et al. Cardiac Dysfunction Among People Living With HIV. JACC Hear Fail. 2019;7:98-108.

3. Bloomfield GS, Leung C. Cardiac Disease Associated with Human Immunodeficiency Virus Infection. Cardiol Clin. 20 17;35:59-70.

4. Sliwa K, Carrington MJ, Becker A, et al. Contribution of the human immunodeficiency virus/acquired immunodeficiency syndrome epidemic to de novo presentations of heart disease in the Heart of Soweto Study cohort. Eur Heart J. 2012;33:866-874.

5. Joint United Nations Programme on HIVIAIDS (UNAIDS). Global HIV and AIDS statistics 2019 Fact sheet. Global HIV \& AIDS statistics - 2019 fact sheet. Accessed April 9, 2020. https://www.unaids.org/en/resources/factsheet

6. Bloomfield GS, Alenezi F, Barasa FA, et al. Human Immunodeficiency Virus and Heart Failure in Low- and Middle-Income Countries. J Am Coll Cardiol Hear Fail. 2016;15:477-491.

7. Agbor V, Essouma M, Ntusi N, et al. Heart failure in sub-Saharan Africa: A contemporaneous systematic review and meta-analysis. Int J Cardiol. 2018;257:207-215.

8. Barbaro G, Klatt E. HIV infection and the cardiovascular system. AIDS Rev. 2002;4:93- 103.

9. Manga P, McCutcheon K, Tsabedze N, et al. HIV and nonischaemic heart disease. J Am Coll Cardiol. 2017;69:83-91.

10. Lang RM, Bierig M, Devereux R, et al. Recommendations for Chamber Quantification: A Report from the American Society of Echocardiography's Guidelines and Standards Committee and the Chamber Quantification Writing Group, Developed in Conjunction with the European Association of Echocardiograph. J Am Soc Echocardiogr. 2005; I 8: I 440- 463.

II. Karavidas A, Xylomenos G, Matzaraki V, et al. Myocardial deformation imaging unmasks subtle left ventricular systolic dysfunction in asymptomatic and treatment-naïve HIV patients. Clin Res Cardiol. 20I 5; 104:975-98I .

12. Geyer H, Caracciolo G, Abe H, et al. Assessment of Myocardial Mechanics Using Speckle Tracking Echocardiography: Fundamentals and Clinical Applications. J Am Soc Echocardiogr. 20 10;23:35 I-369.

13. Blessberger $\mathrm{H}$, Binder $\mathrm{T}$. Two dimensional speckle tracking echocardiography: Basic principles. Heart. 2010;96:716-722

14. Sood V, Jermy S, Saad H, et al. Review of cardiovascular magnetic resonance in human immunodeficiency virus-associated cardiovascular disease. South African J Radiol. 2017;21:1-10.

15. Ferreira VM, Schulz-Menger J, Holmvang G, et al. Cardiovascular magnetic resonance in nonischaemic myocardial inflammation: Expert recommendations. J Am Coll Cardiol. 2018;72:3158-3176.

16. Ridgway JP. Cardiovascular magnetic resonance physics for clinicians: Part I. J Cardiovasc Magn Reson. 2010;12:1-28.

17. Haaf P, Garg P, Messroghli DR, et al. Cardiac TI Mapping and Extracellular Volume (ECV) in clinical practice: A comprehensive review. J Cardiovasc Magn Reson. 2016;18:1-12.

18. Varghese A, Pennell DJ. Cardiovascular magnetic resonance made easy. Churchill Livingstone Elsevier; 2008.

19. Ntusi N, O'Dwyer E, Dorrell L, et al. HIV-I-related cardiovascular disease is associated with chronic inflammation, frequent pericardial effusions, and probable myocardial edema. Circ Cardiovasc Imaging. 20 1 6;9:1 -8.

20. Menacho K, Seraphim A, Ramirez S, et al. Myocardial inflammation and edema in people living with human immunodeficiency virus. JACC CardiovasC Imaging. 2020;1 3:1278-1280.

21. Holloway CJ, Ntusi N, Suttie J, et al. Comprehensive cardiac magnetic resonance imaging and spectroscopy reveal a high burden of myocardial disease in HIV patients. Circulation. 2013; 1 28:8 | 4-822.

22. Hsue PY, Hunt PW, Ho JE, et al. Impact of HIV infection on diastolic function and left ventricular mass. Circ Hear Fail. 20 10;3:132-139.
23. Hulten E, Aslam S, Osborne M, et al. Cardiac sarcoidosis-state of the art review. Cardiovasc Diagn Ther. 2016;6:50-63.

24. Biglands JD, Radjenovic A, Ridgway JP. Cardiovascular magnetic resonance physics for clinicians: Part II. J Cardiovasc Magn Reson. 20 I2; 14:1-40.

25. Lynch J, Hwang J, Bradfield J, et al. Cardiac involvement in sarcoidosis: evolving concepts in diagnosis and treatment. Semin Respir Crit Care Med. 2014:35:372-390.

26. Moon JC, Messroghli DR, Kellman P, et al. Myocardial TI mapping and extracellular volume quantification: A Society for Cardiovascular Magnetic Resonance (SCMR) and CMR Working Group of the European Society of Cardiology consensus statement. J Cardiovasc Magn Reson. 2013;15:92.

27. Vandi G, Calza L, Girometti N, et al. Acute onset myopericarditis as unusual presentation of primary HIV infection. Int J STD AIDS. 2017;28:199-20।.

28. Currie PF, Boon NA. Immunopathogenesis of HIV-related heart muscle disease. AIDS. 2003; 17:S21-S28.

29. Secemsky EA, Scherzer R, Nitta E, et al. Novel biomarkers of cardiac stress, cardiovascular dysfunction, and outcomes in HIV-infected individuals. JACC Hear Fail. 2016;3:591-599.

30. Hsue PY, Waters DD. Heart failure in persons living with HIV infection. Curr Opin HIV AIDS. 2017; 12:534-539.

31. Savvoulidis P, Butler J, Kalogeropoulos A. Cardiomyopathy and Heart Failure in Patients With HIV Infection. Can J Cardiol. 2019;35:299-309.

32. Remick J, Georgiopoulou V, Marti C, et al. Heart failure in patients with human immunodeficiency virus infection: Epidemiology, pathophysiology, treatment, and future research. Circulation. 2014;129:1781- 1789.

33. Luo L, Zeng Y, Li T, et al. Prospective echocardiographic assessment of cardiac structure and function in Chinese persons living with HIV. Clin Infect Dis. 20| 4;58: | 459- | 466.

34. Shaboodien G, Engel ME, Syed FF, et al. The mitochondrial DNA TI6189C polymorphism and HIV-associated cardiomyopathy: A genotype-phenotype association study. BMC Med Genet. 2009; 10:37.

35. Twagirumukiza $M$, Nkeramihigo $E$, Seminega $B$, et al. Prevalence of dilated cardiomyopathy in HIV-infected african patients not receiving HAART: A multicenter, observational, prospective, Cohort Study in Rwanda. Curr HIV Res. 2006;5:129-137.

36. Herskowitz A, Vlahov D, Willoughby S, et al. Prevalence and incidence of left ventricular dysfunction in patients with human immunodeficiency virus infection. Am J Cardiol. 1993;71:955-958

37. Pozzan G, Pagliari C, Tuon FF, et al. Diffuse-regressive alterations and apoptosis of myocytes: Possible causes of myocardial dysfunction in HIVrelated cardiomyopathy. Int J Cardiol. 2009;1 32:90-95.

38. Schuster C, Mayer FJ, Wohlfahrt C, et al. Acute HIV infection results in subclinical inflammatory cardiomyopathy. 2018;218:2-6. 